\title{
Ethanol Production from Low Grade Apples by Using Saccharomyces Cerevisiae
}

\section{Cheema MA, Nadeem F, Akram MS and Saeed M*}

Centre of Agricultural Biochemistry and Biotechnology University of Agriculture, Pakistan

*Corresponding author: Muhammad Saeed, Centre of Agricultural Biochemistry and Biotechnology University of Agriculture, Faisalabad, Pakistan, Tel: 0923027057360; Email: muhammadsaeed7360@gmail.com

\section{Research Article}

Volume 5 Issue 2

Received Date: June 02, 2020

Published Date: July 03, 2020

DOI: 10.23880 /oajmb-16000162

\section{Abstract}

The present research work is important in scenario of energy crises. The aim of this study was to utilize the low grade apples for the production of ethanol which in turn could be used for various purposes. From this study, it is clear that the maximum production of ethanol could be obtained from the apple. In this research maximum ethanol was obtained from low evaluations apples gathered from nearby markets around Lahore. S. cervicieae strain was used for the maximum conversion of sugar contents of apples into ethanol. Various parameters were optimized to get the maximum ethanol production which can be enhanced by further studies and could be beneficial candidate for various applications.

Keywords: Esterification; Oxidation; Dehydrogenation; Dehydration; Basidiomycota; Chytrimycota; Orgosterol; Ethanol

\section{Introduction}

Ethanol is the one of the striking artificial oxygen containing compound. Due to its striking power it has certain properties like fuel, an antifreezer, as a solvent, a beverage, a depressant, germicide and specially its flexibility for intermediate chemical compounds [1]. Due to its dynamic nature, the ethanol under regular condition is a colorless, flammable, volatile and clear liquid. Its aroma is very lovely, familiar and taste pleasant when mixed with water. Due to primarily dependent on the hydroxyl group, the ethanol has both chemical and physical properties.

The group in which hydroxyl group is attached have the different polarity to the molecule which is given hydrogen bonding of intermolecular. In liquid form, the hydrogen bonding is developed by the attraction of hydroxyl to the hydrogen one molecule and second in which hydroxyl to the oxygen molecule. These both type of bonding make the watery alcohol which behaves as though it was mostly dimerized. This type of behavior is equivalent to that water which have strongest bond between them and show in liquid as groups of molecules. The hydroxyl group comprises largely ethanol chemistry. The ethanol chemistry of molecules included the esterification, oxidation, dehydrogenation and dehydration of reactions. In hydroxyl group the hydrogen atom can be changed with different metals. The metals include calcium, potassium and sodium. All these metals are combined to form ethoxide metals with the removal of hydrogen gas [2].

A solution of ethanol which is mostly used in laboratories as disinfectants is 70 to $85 \%$ of ethanol. It destroys the organism by denaturing their structure of protein and dissolving in lipids. It is efficient against different viruses, fungi and bacteria but some time it is ineffective against the spores of bacteria. The alcoholic beverage can be stored for long time due to its disinfectant characteristics [3].

As an alternative of petrol, ethanol is being used as a fuel. The mixture of ethanol with petrol is used to decrease the petroleum consumption. The interest in ethanol production arouse when waste products and agriculture wastes are being employed as substrates for the production of ethanol. Under biological process the bioethanol is produced. Bioethanol is one form of ethanol which is produced from large variety of raw material. There are three main types: the first type is that in which sucrose, which is present in large number of 


\section{Open Access Journal of Microbiology \& Biotechnology}

materials are fermented like sugar cane.

For conventional consumption, the bioethanol has number of advantages. These biofuels are come from renewable sources like crops but not come from finite resources. The other benefits of biofuel from biomass are the less emission of gases because growing fuel crops absorb the $\mathrm{CO}_{2}$ [4]. By growing such crops, the rural economy boosts up through the boosting bioethanol use. The bioethanol is ecological and less harmful then fossils fuels. To fulfil energy needs biofuel production from agricultural waste products plays a major role [5].

There are certain microbial species used in ethanol production including bacteria and fungi. The further bacteria and fungi have types which used in ethanol production. In bacteria the various types of bacteria are used like aerobic bacteria and anaerobic bacteria. The anaerobic bacteria are those which fermented the ethanol in the absences of oxygen. While the aerobic bacteria are those which require the oxygen. The mostly ethanol is produced anaerobically. Similarly, certain types of fungi are used in ethanol production like club fungi (Basidiomycota), breads mold (Zygomycota), yeast and sac fungi (Ascomycota), chutrids (Chytrimycota). The most common fungus is yeast which is Saccharomyces cerevisiae. The Saccharomyces cerevisiae play major role in conversion of apple mixture to ethanol production.

The production of ethanol from the carbon source by $S$. cerevisiae is old method. This method is sparingly important in the field of biotechnology. In the production of all types of ethanol the commonly used yeast play important role. The Saccharomyces cerevisiae is the worldwide and dominating yeast which used in all types of ethanol production. The important characteristics of this yeast are giving aroma and flavor to the different beverages and the important function of yeast is used in fermentation. The pure culture of Saccharomyces cerevisiae is commonly used in large scale beverages production like brewing, whisky production and distilled spirit production.

The growing condition of yeast at small scale and large scale is different. At small scale strains of $S$. cerevisiae can grow in acidic condition and warm condition. At acidic and warm conditions, the temperature is mostly $20^{\circ} \mathrm{C}$ to $30^{\circ} \mathrm{C}$ and $\mathrm{pH}$ is 4.5606 .5 . While at large scale the growth condition of selected strain of yeast is $8^{\circ} \mathrm{C}$ to $15^{\circ} \mathrm{C}$. In the fermentation process of yeast acidify different changes is occurring like proton is secreting the ATP through the action of plasma membrane proton pumping ATPase enzyme and direct secretion of organic chemical acids and in the process the buffering agent is removal.

Under strictly anaerobic condition the yeast cannot grow because sometime strain of $S$. cerevisiae is referred to facultative anaerobes. In the biosynthesis of sterol and membrane fatty acid the oxygen is must require for their growth. Under anaerobic condition the yeast $S$. cerevisiae is auxotrophic of oleic acid and orgosterol. At the time of starting fermentation either some amount of oxygen is required for the growth of fatty acid which is supplemented to the medium of yeast foods [6].

Ethanol productions have many sources. Through fermentation process glucose molecules are breakdown. These glucose molecules are sugars like sugar cane, sugar beets and mostly molasses and starch like corn, wheat and grains and cellulose which is mostly use as raw material. The major five sugars are used like mannose, arabinose, xylose and galactose which contain six carbon sugar [7]. Through coal and gas the synthetic ethanol can be produced from renewable sources. Horticulture crops play vital role in the production of ethanol production. It increase the export quality of horticulture crops but the cost of horticulture is high it is cost effective and time consuming process [8]. In human diet they play important role. The importance of horticulture crops cannot be denied by anyone because these fulfill our need in our diet. Pakistan economy is strong in the sense of fruits production. All types of fruit are produced in the Pakistan. The area covered by the fruits is 776 thousand hectors and annual production of fruits is 6423.9 outputs. In Pakistan the grower's consumers is six to seven intermediate primitive networks.

The genus of apple is Malus and family is Rosacea. This family is very large which included not only the apple but also other types of fruits like palms, raspberries, peaches and pears. There are no grit cells in Malus genus. At the base the styles are united. The fruit is globular and flowers are colored. There are 15 to 20 stamens, two ovules and carpels sepal and petal is five in perfect arrangements. The apple is technically a pome, the tree is mix buds, deciduous and upright speeding [9].

In every year the production of apples in Pakistan is 13 lac 35 thousand tons. Pakistan is world $10^{\text {th }}$ largest country for apple production. The countries which are prominent in apple production are America, Chilly, Italy and France. Their apple production in every year is 50 lac tons. In Pakistan the key contributor of apple is Baluchistan province. While KPK is the $2^{\text {nd }}$ contributor of apple production in Pakistan. Total $25 \%$ of the apples are produced in the KPK. The duration of production of apple is start from December to the end in January. While in Pakistan few farmers cultivate the apples in monsoon weather.

Apple contains large quantity of natural sugars. A large quantity of apples is being discarded annually due to the 


\section{Open Access Journal of Microbiology \& Biotechnology}

low grade in several ways, although these apples contain sugar contents. These sugar contents present in low quality apples cane be utilized for the production of ethanol by the application of $S$. cerevisiae yeast which intern can be used in various applications.

\section{Methodology}

\section{Selection of Materials, Chemicals and Their Maintenance}

Low grade apples and yeast Saccharomyces cerevisiae were selected for the production of ethanol. Low grade apples were purchased from the local market while the Saccharomyces cerevisiae was obtained from the culture bank of Govt. College University, Lahore Pakistan. YPD medium (Yeast extract 1\%, Peptone 2\%, Tryptophan $0.033 \%$, Dextrose 2\%) was used for the culturing of S. cerevisiae. A single colony of $S$. cerevisiae was streaked on the YPD-agar plate in biosafety cabinet and incubated at $30^{\circ} \mathrm{C}$ overnight with upside down.

\section{Inoculum Preparation}

A fresh inoculum was prepared from the overnight grown culture of $S$. cerevisiae in the above mentioned YPD broth for the production of ethanol from low quality apples. A single colony of overnight grown culture of $S$. cerevisiae was aseptically transferred in the freshly prepared YPD broth under aseptic conditions and incubated at $30^{\circ} \mathrm{C}$ until its optical density reached to 0.8 at $600 \mathrm{~nm}$.

\section{Fermentation}

Surface culture fermentation technique was employed for the production of ethanol from low quality apples. For the production of ethanol following steps were performed:

\section{Preparation of Apple Extract}

For the preparation of apple extract, $500 \mathrm{~g}$ of low quality apples were weighed and made their extract by adding 1000 $\mathrm{ml}$ of distilled water with the help of blender. A homogenized mixture of apple was prepared. Apple's juice was filtered with muslin cloth and residue was again grinded and extract was made up to the point, where no further extract was possible. After checking the total sugar contents, mixture was sterilized by autoclaving under specific conditions. After cooling, it was further used for inoculation of yeast.

\section{Inoculation of $S$. cerevisiae}

Freshly prepared inoculum of S. cerevisiae was inoculated into the sterilized apple mixture under aseptic conditions. Flasks were cotton lugged and incubated at $30^{\circ} \mathrm{C}$ in a static incubator.

\section{Determination of Ethanol and Sugar Contents}

Qualitative measurement of the production of ethanol was done periodically after 24, 48, 72, 96 and 120 hours with the help of alcoholmeter while the quantitative measurement was carried out by dichromate method. Reduction in sugar contents was also calculated by refractometer as well as by DNS method (Miller, 1959) after every 24 hours until all sugar contents were consumed.

\section{Optimization of Ethanol Production}

Production of ethanol from low quality apples was optimized by optimizing following parameters:

\section{Effect of Incubation Temperature}

Effect of incubation temperature upon production of ethanol was checked by supplying different incubation temperatures ranging from $20^{\circ} \mathrm{C}$ to $40^{\circ} \mathrm{C}$. Ethanol production and sugar consumption was calculated after 72 hours by dichromate method and DNS method, respectively.

\section{Effect of pH on the Production of Ethanol}

Effect of $\mathrm{pH}$ on the production of ethanol from low quality apples was determined by setting the $\mathrm{pH}$ of the apple extract ranging from 2 to 7 . After inoculation with yeast inoculum all flasks were incubated at $30^{\circ} \mathrm{C}$ for 72 hours and then ethanol production and sugar consumption was carried out by standard methods.

\section{Effect of Incubation Time on Ethanol Production}

To determine the effect of incubation time on the production of ethanol, after inoculation of yeast, the apple extract was incubated for different time periods ranging from 24 to 120 hours at $30^{\circ} \mathrm{C}$. The ethanol production and reduction of sugar contents were determined by standard methods as mentioned above.

\section{Effect of Inoculum Size on Ethanol Production}

To determine the effect of inoculum size on the production of ethanol from low quality apples, a range of inoculum sizes was used (1-5\%) for inoculation of apple extract. Incubation was carried out at $30^{\circ} \mathrm{C}$ for 72 hours. Production of ethanol and reduction in sugar contents were calculated as mentioned above. 
Results

\section{Culturing of $S$. cerevisiae on YPD-Agar Medium}

Yeast strain of S. cerevisiae was cultured on YPD-agar medium to obtain the fresh culture. Culture was streaked on freshly prepared YPD-agar plates and incubated overnight at $30^{\circ} \mathrm{C}$. The results obtained are presented in Figure 1.

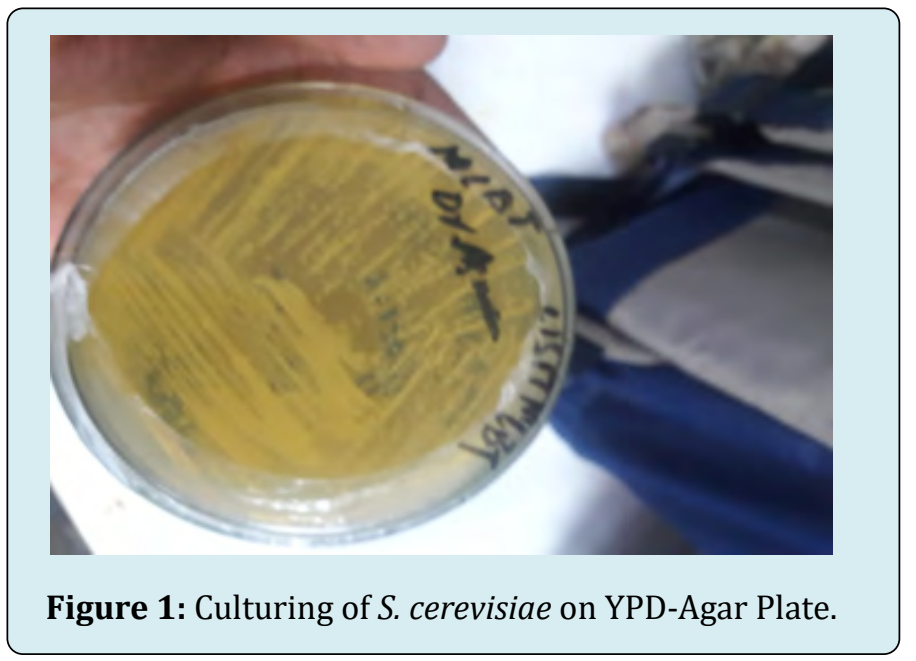

\section{Ethanol Production from Low Quality Apples}

Apple extract of low quality apples was prepared, sterilized and inoculated with $S$. cerevisiae for the production of ethanol. Following parameters were optimized for the maximum production of ethanol from low quality apples:

\section{Effect of Time of Incubation on Production of Ethanol}

Impact of incubation time on the ethanol production from low quality apples by the application of $S$. cerevisiae, the cultured broth was checked after 24 hours, $48 \mathrm{~h}, 72 \mathrm{~h}, 96 \mathrm{~h}$ and $120 \mathrm{~h}$, for 24 hours for ethanol production along with reduction in sugar contents. Results obtained indicated that maximum ethanol (13\%) was produced after 72 hours of incubation at $30^{\circ} \mathrm{C}$ with minimum sugar contents $(0.247 \%)$ as shown in Figure 2. At other duration of time like 24 hours, 48 hours, 96 hours and 120 hours, the production of ethanol was $9.40 \%, 12 \%, 9.73 \%$, and $7 \%$ with sugar contents $8.25 \%$, $3.14 \%, 0.214 \%, 0.147 \%$, respectively as shown in Figure 2.

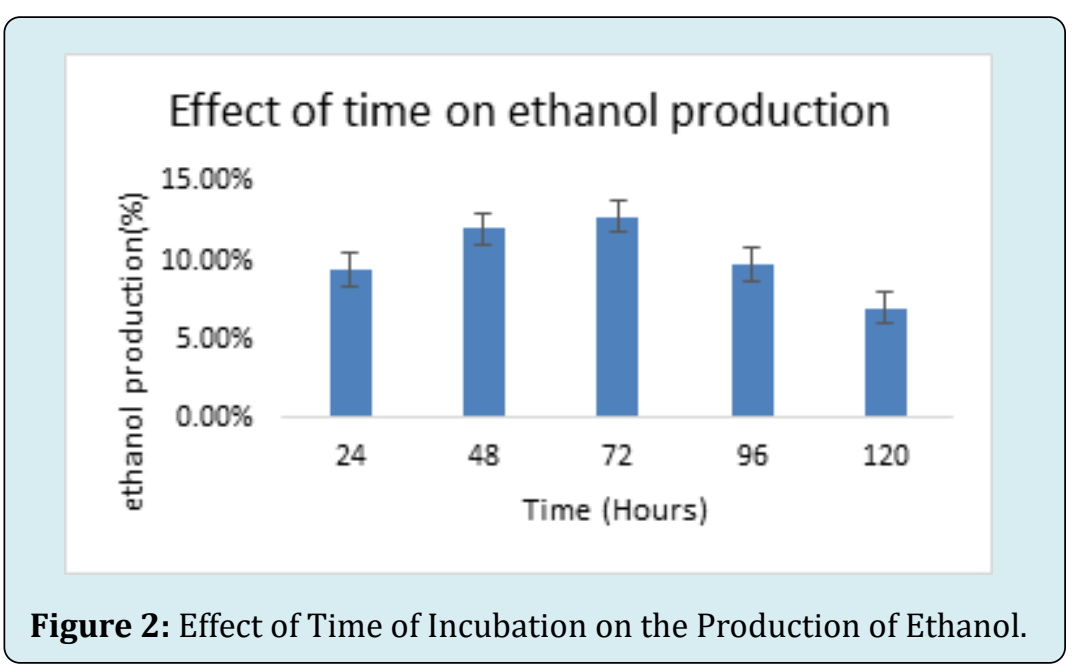

\section{Effect of Temperature}

To determine the effect of temperature on the production of ethanol from low quality apples by using $S$. cerevisiae, the inoculated apple extract was kept with the initial sugar concentration of $20 \%$ on $20^{\circ} \mathrm{C}, 25^{\circ} \mathrm{C}, 30^{\circ} \mathrm{C}, 35^{\circ} \mathrm{C}, 37^{\circ} \mathrm{C}$ and $40^{\circ} \mathrm{C}$. The estimations were carried out after 72 hours of incubation. Results obtained indicated that maximum ethanol (10.5\%) 
was produced at $30^{\circ} \mathrm{C}$ with minimum sugar concentrations $(0.675 \%)$ as shown in Figure 3. At other temperatures like $20^{\circ} \mathrm{C}, 25^{\circ} \mathrm{C}, 35^{\circ} \mathrm{C}, 37^{\circ} \mathrm{C}$ and $40^{\circ} \mathrm{C}$, the production of ethanol was $3.4 \%, 6.7 \%, 8.2 \%, 7.2 \%$ and $5 \%$ with sugar remnants as $15.78 \%, 11.78 \%, 0.42 \%, 0.35 \%$, respectively as shown in Figure 3.

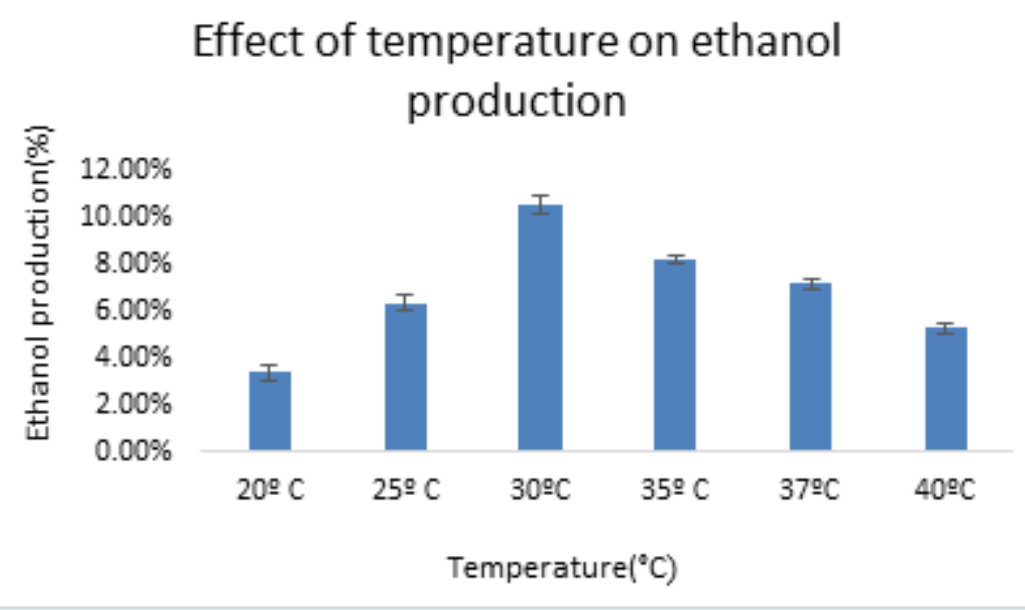

Figure 3: Effect of Incubation Temperature on Ethanol Production.

\section{Effect of pH}

To determine the effect of $\mathrm{pH}$ on the production of ethanol from low quality apples by $S$. cerevisiae, a range of $\mathrm{pH}$ (2.07.0) was selected for the raw apple extract and production of ethanol was estimated after 72 hours of incubation at $30^{\circ} \mathrm{C}$. Results obtained indicated that maximum ethanol $(12 \%)$ was produced at pH 5.0 as shown in Figure 4. At other $\mathrm{pH}$ values like $2.0,3.0,4.0,6.0$ and 7.0 , the production of ethanol was $2.40 \%, 5.53 \%, 10.40 \%, 9 \%$ and $7.2 \%$ with sugar concentrations $16.0 \%, 12.74 \%, 9.87 \%, 10.82 \%$ and $14.02 \%$, respectively as represented in Figure 4.

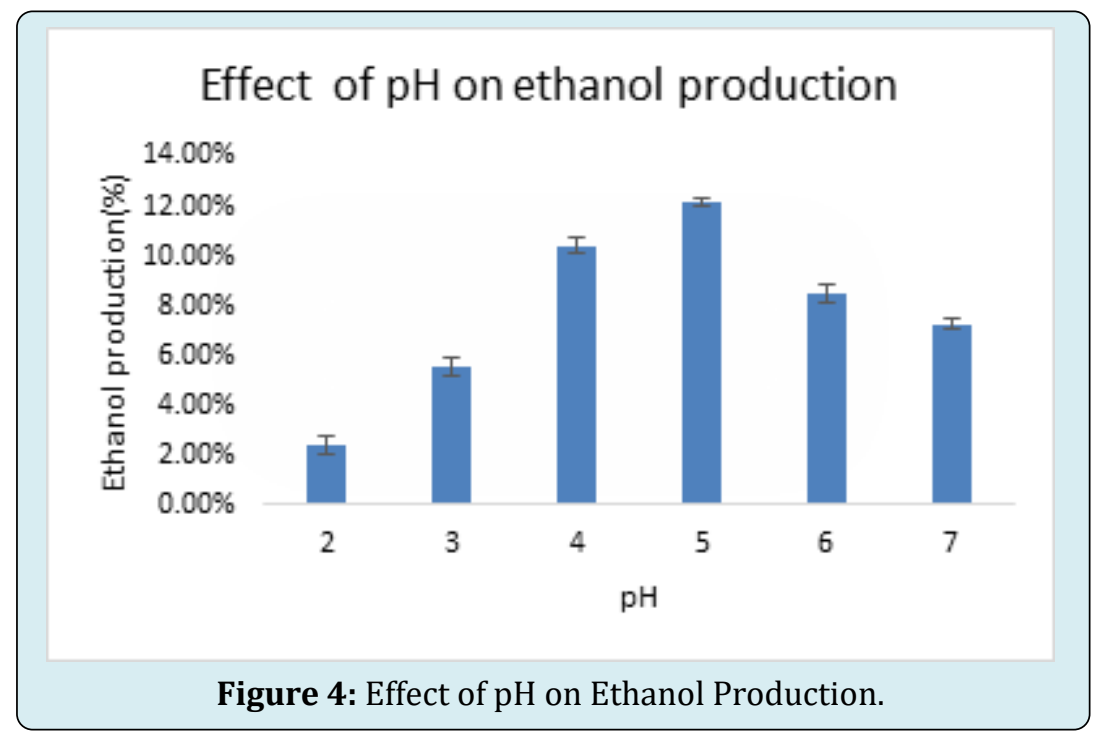

\section{Effect of Different Inoculum Size}

To see the impact of the inoculum size on the ethanol production, the apple extract was inoculated with different percentages of yeast inoculum like, $1 \%, 2 \%, 3 \% 4 \%$ and $5 \%$. The estimations were carried out after 72 hours of incubation at $30^{\circ} \mathrm{C}$ with initial pH 5.0 . Results obtained indicated that maximum ethanol (15.3\%) was produced with $4 \%$ inoculum size with minimum sugar concentration $(0.32 \%)$ as shown in Figure 5. At other inoculum sizes like 1\%, 2\%, 3\% and $5 \%$, the production of ethanol was $9.4 \%, 12.3 \%, 13.3 \%$, and $14.3 \%$ having sugar contents $8.25 \%, 6.68 \%, 4.31 \%$ and $2.17 \%$, respectively as shown in Figure 5. 


\section{Open Access Journal of Microbiology \& Biotechnology}

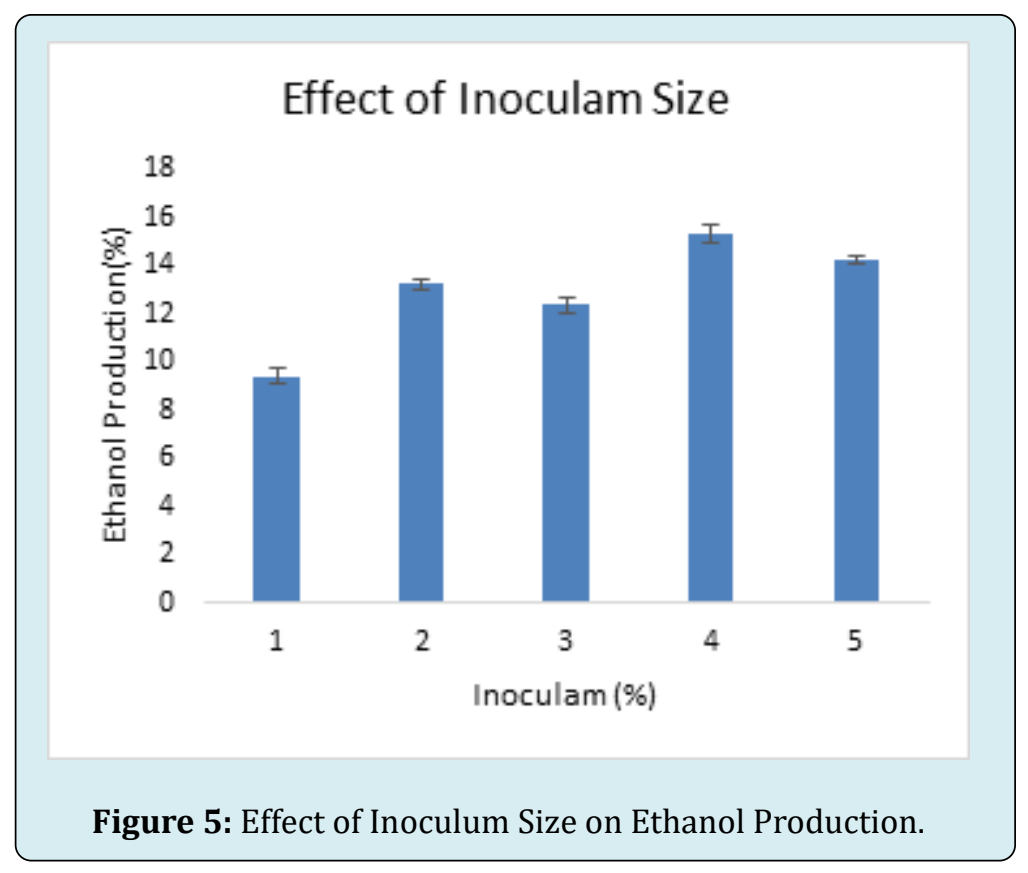

\section{Discussion}

Ethanol is a biofuel which can be obtain by the fermentation of sugar by $S$. cerevisiae. In the present study ethanol fermentation was carried out from low grade apples by the application of $S$. cerevisiae. The results obtained in this study showed excellent potential for residue-based ethanol production. Furthermore, ethanol production from the apples residues can be analyzed under the concept of market view. This work is based on waste products of apples which were converted in to ethanol. These residues of apples contain more sugar concentration and which make the more ethanol. The initial concentration of sugar contents is greater than work of researchers. Various parameters were optimized for the best production of ethanol from low quality apples by yeast including effect of temperature, $\mathrm{pH}$, time of incubation and inoculum size. In the effect of incubation temperature is important factor for the ethanol production. We got the minimum results at $20^{\circ} \mathrm{C}$ while in other research works obtained the minimum results at $25^{\circ} \mathrm{C}$. At $30^{\circ} \mathrm{C}$ ethanol yield was most efficient and calculated as $10 \%$ as shown in Figure 4. Essentially $\mathrm{pH}$ is vital for the ethanol production. Maximum ethanol production was achieved at $\mathrm{pH} 5.0$ as shown in Figure 4. Low and high $\mathrm{pH}$ resulted in inhibition of yeast growth which intern effected the production of ethanol. Yadav, et al. [10] obtained the maxim ethanol production at pH 6.0.

Another factor which effects the production of ethanol is time duration. The time is important factor for the conversion of apples contents in to alcohol contents. A low ethanol product is obtained after 120 hours and maximum results were obtained after 72 hours. Gough, et al. obtained the maximum production of ethanol after 48 hours.

The important last but not the least factor which effect the production of ethanol is inoculum size. If the size of Inoculum is increase then production of ethanol is also increase. In fermentation process of ethanol, the size of inoculum has great importance. For inoculate the flasks different size of inoculum 1-5\% (v/v) were used. There is direct relation between inoculum size and amount of ethanol. The quantity of ethanol produced regularly increased if the size of inoculum is increase. But the maximum amount of ethanol is production is $15 \%$ at inoculum size $4 \%$.Further if the size of inoculum is increase don't result in considerable development of ethanol production. This finding is in agreement with the other researchers [11-14].

\section{Conclusion}

The present research work is important in scenario of energy crises. The aim of this study was to utilize the low grade apples for the production of ethanol which in turn could be used for various purposes. From this study, it is clear that the maximum production of ethanol could be obtained from the apple. In this research maximum ethanol was obtained from low evaluations apples gathered from nearby markets around Lahore. S. cervicieae strain was used for the maximum conversion of sugar contents of apples into ethanol. Various parameters were optimized to get the maximum ethanol production which can be enhanced by further studies and could be beneficial candidate for various applications. 


\section{Open Access Journal of Microbiology \& Biotechnology}

\section{References}

1. (1980) US Department of Energy, Fuel from farms. U.S. Department of Energy, Oak Ridge, Tenn.

2. Um BH (2007) Optimization of ethanol production from concentrated substrate (Doctoral dissertation).

3. Eddaoudi M, Moler DB, Li H, Chen B, Reineke TM, et al. (2001) Modular chemistry: secondary building units as a basis for the design of highly porous and robust metalorganic carboxylate frameworks. Acc Chem Res 34(4): 319-330.

4. Tyson KS, Riley CJ, Humphreys KK (1993) Fuel Cycle Evaluations of Biomass Ethanol and Reformulated Gasoline. National Renewable Energy Laboratory (NREL), Golden, CO, NREL/TP-463-4950.

5. Wyman CE (1994) Ethanol from Lignocellulosic Biomass: Technology, Economics, and Opportunities. Bioresource Technology 50(1): 3-15.

6. Walker GM, Stewart GG (2016) Saccharomyces cerevisiae in the production of fermented beverages. Beverages 2(4): 30 .

7. Wyman CE (1996) Handbook on Bioethanol: Production and Utilization. Taylor and Francis, Washington, DC.
8. Kondal K (2014) Growth rate of area, production and productivity of onion crop in Andhra Pradesh. Indian Journal of Applied Research 4(3): 4-6.

9. Gao YR, WU D (2005) Study on Production Technology of Apple Wine [J]. Liquor-making Science \& Technology 2: 020 .

10. Yadav A, Dilbaghi N, Sharma S (1997) Pretreatment of sugarcane molasses for ethanol production by yeast. Indian Journal of Microbiology 37(1): 37-40.

11. Bajaj KB, Yousef S, Thakur LR (2001) Selection and characterization of yeasts for desirable. Indian Journal of Microbiology 41(2): 107-110.

12. Nowak J (2001) Comparisons of polish industrial distillery yeast with ethanol producing bacteria Zymomonas mobilis. Elec J Pol Agr Univ 4: 2.

13. Kordowska-Waiter M, Zdzissla T (2001) Ethanol production on the media containing glucose and xylose by coculture of Pichia stipitis ccy 39501 and respiratory deficient mutant of Saccharomyces cerevisiae. V Elec J Pol Agr Univ 4: 15.

14. Alegre RM, Rigo M, Jokes I (2003) Ethanol fermentation of a diluted molasses medium by saccharomyces cerevisiae immobilized on chrysotile. Braz Arch Biol Technol 46(4). 